\title{
Impact of growth and uptake patterns of arbuscular mycorrhizal fungi on plant phosphorus uptake - a modelling study
}

\author{
Andrea Schnepf • Tiina Roose • Peter Schweiger
}

Received: 15 December 2007 / Accepted: 7 August 2008/Published online: 3 September 2008

(C) The Author(s) 2008. This article is published with open access at Springerlink.com

\begin{abstract}
In this paper we present a mathematical model for estimating external mycelium growth of arbuscular mycorrhizal fungi and its effect on root uptake of phosphate (P). The model describes $\mathrm{P}$ transport in soil and $\mathrm{P}$ uptake by both root and fungi on the single root scale. We investigate differences in soil $\mathrm{P}$ depletion and overall $\mathrm{P}$ influx into a mycorrhizal root by assuming that different spatial regions of mycelia are active in P uptake. When all external hyphae contribute to $\mathrm{P}$ uptake, overall uptake is dominated by the fungus and the most effective growth pattern appears to be the one using a high level of anastomosis. The same is true when only the proportion of external hyphae assumed to be active contributes to uptake. When uptake is restricted to the tips, hyphal contribu-
\end{abstract}

Responsible Editor: Petra Marschner.

A. Schnepf $(\bowtie) \cdot$ P. Schweiger

Department of Forest and Soil Sciences, Institute of Soil Science,

BOKU-University of Natural Resources

and Applied Life Sciences,

Peter Jordan Strasse 82,

1190 Vienna, Austria

e-mail: andrea.schnepf@boku.ac.at

T. Roose

Mathematical Institute,

Oxford Centre for Industrial and Applied Mathematics

and Centre for Mathematical Biology,

University of Oxford,

24-29 St Giles',

Oxford OX1 3LB, UK tion to overall $\mathrm{P}$ uptake is less dominant; the most effective growth pattern appears to be the one characterised by nonlinear branching where branching stops at a given maximal hyphal tip density. Comparison to measured $\mathrm{P}$ depletion in the literature suggests that the scenario where active hyphae are contributing to $\mathrm{P}$ uptake is likely to fit the data best. These quantitative predictions promote our understanding of the mycorrhizal symbiosis and its role in plant $\mathrm{P}$ nutrition.

Keywords Arbuscular mycorrhiza $\cdot$ Fungal growth . Mathematical model · Plant phosphorus uptake

\section{Introduction}

Phosphorus is one of the essential mineral nutrients for plants. The preferred form of phosphorus taken up by plants is ortho-phosphate ${ }^{1}$ (Vance 2003). Due to the fact that phosphate $(\mathrm{P})$ is generally not very mobile in soils, narrow depletion zones in the order of millimetres form around P-absorbing roots (Hinsinger et al. 2005). Low phosphate concentrations in soil can be limiting for plant growth. Therefore, plants have developed mechanisms, such as symbiotic relations with soil fungi, to increase their access to soil phosphate. Probably the most important symbiotic

\footnotetext{
${ }^{1}$ Under nonreductive conditions, inorganic phosphorus in soils predominantly occurs in the form of ortho-phosphate (Gisi et al. 1997). In the following, we will use the term phosphate to refer to ortho-phosphate and its protonated forms.
} 
mechanism is the formation of mycorrhizas, mutualistic symbiotic associations between plant roots and specific soil fungi. Arbuscular mycorrhizas are the most widespread type of mycorrhiza formed by the majority of crop plant species and more than $70 \%$ of all terrestrial plants (Brundrett 2002). In the current paper, only arbuscular mycorrhizal associations are examined. In addition to the direct uptake pathway via roots, arbuscular mycorrhizal (AM) fungi provide plants with an additional pathway for $\mathrm{P}$ uptake. They are able to deliver $\mathrm{P}$ which would otherwise not be directly accessible for the plant (Zhu et al. 2001). This pathway includes $\mathrm{P}$ uptake from soil by the external fungal mycelium, translocation towards the root surface, and transfer into the root cortical cells. In rhizosphere models, $\mathrm{P}$ uptake from soil by both roots and fungi is usually described by Michaelis-Menten kinetics (Barber 1995; Schweiger and Jakobsen 1999). However, uptake can be more complex, following two independent Michaelis-Menten type systems, i.e., a high and a low-affinity transport mechanism. For some plant species, the presence of up to seven transport kinetics has been suggested and the molecular and biochemical characterisation of the corresponding $\mathrm{P}$ transport systems is currently extensively studied (Bucher 2007; Raghothama and Karthikeyan 2005).

Model calculations have shown that the uptake properties of the external hyphae as well as the dynamics of the growth of external fungal mycelium can potentially account for most of the plant $\mathrm{P}$ uptake (Schnepf and Roose 2006). This confirmed experimental data, in which arbuscular mycorrhizal fungi were found to dominate plant P supply (Smith et al. 2003). The model presented by Schnepf and Roose (2006) quantifies the contribution of arbuscular mycorrhizal fungi with a linear branching growth pattern (where branching is linearly proportional to the hyphal tip density) to plant $\mathrm{P}$ nutrition. They concluded that uptake of $\mathrm{P}$ occurs mainly at the front of the growing fungal mycelium and that translocation within the fungal mycelium is so fast that $\mathrm{P}$ availability within the fungus is not a limiting step in plant $\mathrm{P}$ acquisition. By means of a fungal growth model, Schnepf et al. (2007) assigned different growth patterns to three arbuscular mycorrhizal fungi, i.e. Scutellospora calospora isolate WUM 12(2), Glomus sp. isolate WUM 10(1) and Acaulospora laevis isolate WUM 11(4) which were examined in a study of Jakobsen et al. (1992a). The hyphal length and tip densities resulting from calibrating the model of Schnepf et al. (2007) to the measured hyphal length densities are shown in Figs. 4 and 5, respectively. The fungal growth processes apical branching (tip splitting), tip-hyphae anastomosis and tip-tip anastomosis which account for the different fungal growth patters used in the model are depicted in Fig. 1.

Scutellospora calospora WUM 12(2) appeared to receive a lot of support from the root-soil boundary for the creation of new hyphal tips, and it produced the largest biomass of all fungal species considered. By support we mean the supply of carbon or energy from inside the root to the fungus. The growth pattern of this fungus was called linear branching pattern as the rate of tip splitting was linearly proportional to the hyphal tip density. Glomus sp. WUM 10(1) was found to be described best by nonlinear branching where branching ceases at a maximal hyphal tip density, possibly due to competition for resources. The main pattern of Acaulospora laevis WUM 11(4) was considered to be tip-hyphae anastomosis, characterised by the formation of an interconnected network. The most distinct feature of this fungal species was the presence of a peak of hyphal length density at the front of the colony (Jakobsen et al. 1992a; Schnepf et al. 2007). All fungal species grew up to ten centimetres away from the root surface within 47 days. Thus, the external fungal hyphae provided the plants with the possibility to exploit a large soil volume they could not access on their own. It is the goal of this paper to investigate the impact of the different AM fungal growth patterns on plant $\mathrm{P}$ nutrition. In particular, we will show the differences in soil exploitation and in overall $\mathrm{P}$ influx into a mycorrhizal root which is colonised by AM fungi with different growth patterns.

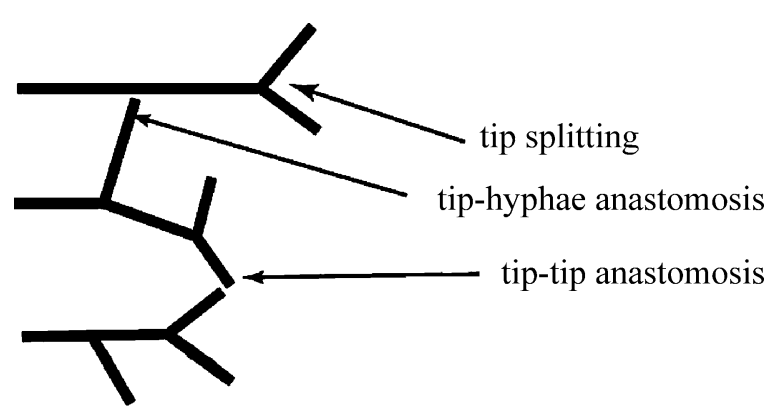

Fig. 1 Schematic representation of the fungal growth processes, apical branching (tip splitting), tip-hyphae anastomosis and tip-tip anastomosis, included in the hyphal growth model of Schnepf et al. (2007) 
Increased $\mathrm{P}$ uptake by arbuscular mycorrhizal plants is generally attributed to the increased surface area available for uptake. However, it is less clear exactly how large this increased surface area for uptake is. It is often assumed that AM fungal hyphae take up nutrients along their entire length (Schweiger and Jakobsen 1999). However, experimental results have led some authors (Boddington and Dodd 1999; Ezawa et al. 2003) to distinguish between a metabolically active and inactive part of the mycelium, implying that only the active part is contributing to P uptake. Also morphological studies of the external mycelium of AM fungi have led some authors to suggest that only a part of the mycelium is acquiring P (Bago 2000). The results from another study again (Jolicoeur et al. 1998) suggest that hyphal $\mathrm{P}$ uptake only occurs in a small region behind the hyphal tips. With our model, we test these hypotheses on mycorrhizal uptake patters for all three fungal growth patterns and quantify how they are expected to influence the plant $\mathrm{P}$ uptake. Furthermore, we assess differences in spatial $\mathrm{P}$ acquisition and how they are related to gross structural features such as hyphal length densities.

\section{Model development}

Motivating experiment and choice of model geometry

Li et al. (1991a) have shown that arbuscular mycorrhizal plants can deplete the soil for P more than 10 $\mathrm{cm}$ away from the root surface. In their experiment, they grew plants in special pots were hyphal compartments were separated from the main root compartment by a membrane that only hyphae, but not roots, could penetrate (see Fig. 2).

This experimental setup motivates the choice of geometry for our model. When the roots in the root compartment are very dense, the dynamics of root growth within this compartment can be neglected and the membrane can be regarded as the root-soil boundary. One-dimensional Cartesian coordinates are appropriate for this system since changes in P concentration and hyphal length densities are only measured with respect to their distance from the planar root-soil boundary.

We use the model that was first described in Schnepf et al. (2007) to describe AM fungal growth. The creation of a $\mathrm{P}$ sink term based on the fungal growth model and the uptake capacity of the external hyphae is described in Schnepf and Roose (2006). This paper combines and extends the work of these two previous publications and examines how different growth and uptake patterns of AM fungi are expected to affect the total $\mathrm{P}$ uptake of a mycorrhizal root.

Model for soil $\mathrm{P}$ concentration near a mycorrhizal root

The model for $\mathrm{P}$ concentration in the soil near a mycorrhizal root is described in Schnepf and Roose (2006) and will briefly be described in the following section. The model equations are given by

$$
\begin{aligned}
& (b+\theta) \frac{\partial c}{\partial t}=D \theta f \frac{\partial^{2} c}{\partial x^{2}}+q \frac{\partial c}{\partial x}-F_{\mathrm{myc}}(\rho, n, x, t), \\
& D \theta f \frac{\partial c}{\partial x}+q c=\frac{F_{\mathrm{m}} c}{K_{\mathrm{m}}+c}, \text { at } x=0, \\
& c=c_{0}, \text { as } \quad x \rightarrow \infty
\end{aligned}
$$

$c=c_{0}$, at $t=0$,

where $x$ is the distance from the root surface, $t$ is time, $b$ is the soil buffer power, $\theta$ is the volumetric water content, $f$ is the impedance factor, $D$ is the diffusion coefficient in free solution, $q$ is the Darcy flux of water towards the root surface, $F_{\mathrm{m}}$ is the maximal influx of solute into root, $K_{\mathrm{m}}$ is the Michaelis-Menten constant of the root, $c_{0}$ is the initial solute concentration in soil, $\rho$ is the hyphal length density, $n$ is the hyphal tip density, and $F_{\text {myc }}$ is the volumetric sink term that represents solute uptake by the fungal mycelium. The expression for $F_{\text {myc }}$ is based on a model for the growth of the external mycelium as well as on a model for P uptake by individual hyphae. Due to the low mobility of $\mathrm{P}$ in soils, depletion zones around single hyphae may in some cases develop similar to the depletion zones created around roots. However, Schnepf and Roose (2006) found that the depletion zone around a single hypha is negligibly small when hyphal uptake is small compared to diffusion in soil. This is the case when $\frac{F_{\mathrm{m}, \mathrm{h}}}{K_{\mathrm{m}, \mathrm{h}}} \ll \frac{D \theta f}{r_{\mathrm{h}}}$, assuming that the parameters describing diffusion, 
Fig. 2 Schematic representation of the experimental setup that motivates the model presented in this study (after Li et al. (1991a))

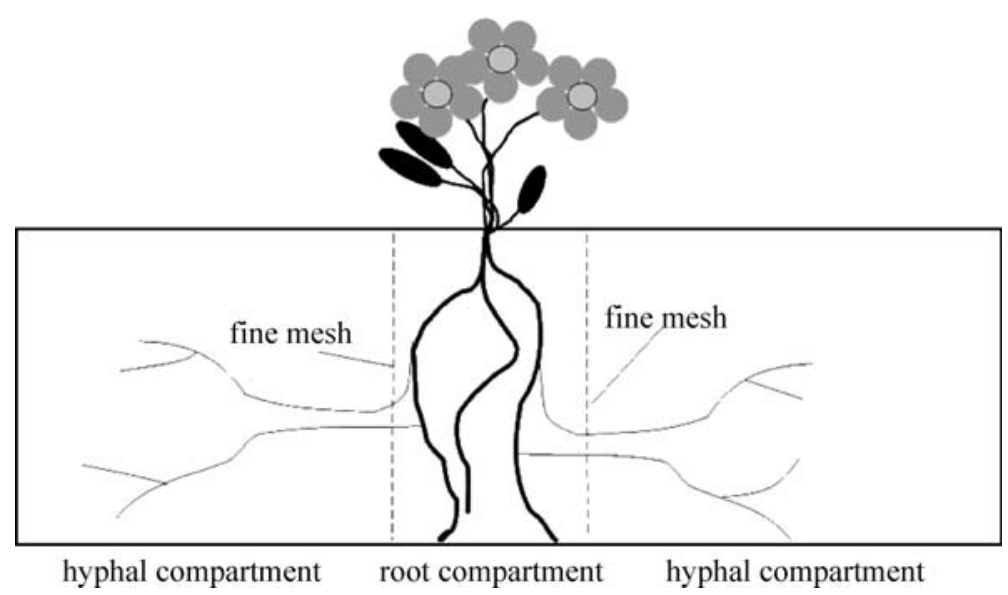

hyphal uptake and hyphal radius are constant. This condition holds for given soil and fungal uptake properties as long as the hyphal radius $r_{\mathrm{h}}$ is smaller then $\frac{D \theta f K_{\mathrm{m}, \mathrm{h}}}{F_{\mathrm{m}, \mathrm{h}}}$. Because of the small radius $r_{\mathrm{h}}$ of a hypha which is in the order of micrometers, this will be true for most fungal species in soil. For example, if we consider the soil and fungal uptake properties used in this study (see Tables 1 and 2 below), the hyphal radius would have to be bigger than $22 \mu \mathrm{m}$ for this condition not to hold. Therefore, in the current study, we will assume that the hyphal radius is smaller than $22 \mu \mathrm{m}$ and that the concentration gradients around hyphae are negligibly small. Under the further assumption that hyphae can take up $\mathrm{P}$ along their entire length, Schnepf and Roose (2006) showed that in this case the sink term $F_{\text {myc }}$ can be expressed as

$F_{\text {myc }}(x, t, \rho, n, c)=2 \pi r_{\mathrm{h}} \rho(x, t) \frac{F_{\mathrm{m}, \mathrm{h}} c(x, t)}{K_{\mathrm{m}, \mathrm{h}}+c(x, t)}$,

where $F_{\mathrm{m}, \mathrm{h}}$ is the maximal influx into hyphae, $K_{\mathrm{m}, \mathrm{h}}$ is the Michaelis-Menten constant of hyphae, $c$ is the local concentration of solutes in soil, $\rho$ is the local hyphal length density, and thus $2 \pi r_{\mathrm{h}} \rho$ is the total surface area of extraradical hyphae. Modifications of Eq. 5 will be related to hyphal growth and uptake patterns, and the effect of this on overall $\mathrm{P}$ influx into a mycorrhizal root will be investigated.

Fungal growth model

The evolution and distribution of the hyphal mycelium in terms of the hyphal length and tip densities is given in Schnepf et al. (2007) and will briefly be described in the following section. From this model, we gain the expression for $\rho(x, t)$ required in Eq. 5 . Hyphal tips move through the soil due to elongation of the zone just behind the tips. The hyphal length density $\rho$ is regarded as the trail left behind by the tips, decreased by hyphal death. New tips are created by apical branching and the number of tips is reduced by tip death, tip-tip and tip-hypha anastomosis. Since arbuscular mycorrhizal fungi are obligate symbionts and are always attached to the root, we need to take

Table 1 Parameter values for P transport model

\begin{tabular}{lllll}
\hline Symbol & Name & Units & Value & Source \\
\hline$D$ & Diffusion coefficient in free solution & $\mathrm{cm}^{2} \mathrm{~s}^{-1}$ & $10^{-5}$ & Lide (2000) \\
$\theta$ & Volumetric water content & $\mathrm{cm}^{3} \mathrm{~cm}^{-3}$ & 0.3 & Barber (1995) \\
$f$ & Impedance factor & $\mathrm{cm}^{3} \mathrm{~cm}^{-3}$ & 0.308 & $f=1.6 \theta-0.172($ Barber 1995) \\
$q$ & Darcy flux & $\mathrm{cm} \mathrm{s}^{-1}$ & $10^{-7}$ & Barber (1995) \\
$b$ & Buffer power & $\mathrm{cm}^{3} \mathrm{~cm}^{-3}$ & 239 & Barber (1995) \\
$c_{0}$ & Initial concentration of P in solution & $\mu \mathrm{mol} \mathrm{cm}^{-3}$ & $10^{-4}$ & Barber (1995) \\
$F_{\mathrm{m}}$ & Maximal influx of P into root & $\mu \mathrm{mol} \mathrm{cm}^{-2} \mathrm{~s}^{-1}$ & $3.26 \times 10^{-6}$ & Tinker and Nye (2000) \\
$K_{\mathrm{m}}$ & Michaelis-Menten constant for root P uptake & $\mu \mathrm{mol} \mathrm{cm}$ & $5.8 \times 10^{-3}$ & Tinker and Nye (2000) \\
\hline
\end{tabular}


Table 2 Parameter values for hyphal uptake

\begin{tabular}{lllrl}
\hline Symbol & Name & Units & Value & Source \\
\hline$r_{\mathrm{h}}$ & Hyphal radius & $\mathrm{cm}$ & $5.0 \times 10^{-4}$ & Ezawa et al. (2002) \\
$F_{\mathrm{m}, \mathrm{h}}$ & Maximal influx of P into hypha & $\mu \mathrm{mol} \mathrm{cm}^{-2} \mathrm{~s}^{-1}$ & $3.26 \times 10^{-6}$ & Same values as for root \\
$K_{\mathrm{m}, \mathrm{h}}$ & Michaelis-Menten constant for hyphal P uptake & $\mu \mathrm{mol} \mathrm{cm}$ & $5.8 \times 10^{-3}$ & Same values as for root \\
\hline
\end{tabular}

the effect of the root-soil boundary into account. In this paper we assume that hyphal tips appear at the root-soil boundary and that the number of tips increases linearly with time. This hyphal population growth model has macroscopic outputs (hyphal length and tip densities), but it is based on average properties of the individual hyphae. The equations describing fungal growth are given by

$\frac{\partial \rho}{\partial t}=v n-d \rho$,

$$
\begin{aligned}
\frac{\partial n}{\partial t}= & -v \frac{\partial n}{\partial x}+b_{n}\left(1-\frac{n}{n_{\max }}\right)-d_{n} n-a_{1} n \rho \\
& -a_{2} n^{2},
\end{aligned}
$$

$n=a t+n_{0, b}, \quad$ at $\quad x=0, t>0$,

$\rho=n=0, \quad$ at $\quad t=0$,

where $v$ is the tip elongation rate, $d$ is the hyphal death rate, $b_{n}$ is the tip branching rate, $n_{\max }$ is the maximal tip density, $d_{n}$ is the tip death rate, $a_{1}$ is the tip-hypha anastomosis rate, $a_{2}$ is the tip-tip anastomosis rate, $a$ is the proliferation parameter for the hyphal tip density at the root-soil boundary, and $n_{0, b}$ is the initial tip density at the root-soil boundary.

\section{Adaptations to the sink term $F_{\text {myc }}$}

In this paper, we will explore the effect of three different scenarios of hyphal uptake patterns and three different fungal growth patterns on overall $\mathrm{P}$ influx into a mycorrhizal root. The different fungal growth patterns are due to different parameter values in the fungal growth model and no changes of the model equations are required. Consideration of uptake patterns other than the one where the whole length of extraradical hyphae are capable of taking up P requires adaptations to the sink term $F_{\text {myc }}$ given by Eq. 5. According to the different assumptions with regard to which parts of the mycelium are contributing to $\mathrm{P}$ uptake as described in the introduction, we will consider three different uptake scenarios. "Full length uptake" is based on the condition that hyphae are capable of taking up phosphate along their entire length so that $F_{\text {myc }}$ is given by Eq. 5. The "tip uptake scenario" assumes that hyphal $\mathrm{P}$ uptake occurs only along a defined length of hypha at and behind the tips. Therefore, we take $F_{\text {myc }}$ to be proportional to the density of the tips, and the sink term $F_{\text {myc }}$ becomes

$F_{\text {myc }}(x, t, \rho, n, c)=2 \pi r_{\mathrm{h}} z n(x, t) \frac{F_{\mathrm{m}, \mathrm{h}} c(x, t)}{K_{\mathrm{m}, \mathrm{h}}+c(x, t)}$,

where $z$ is the length of the zone behind the tip where uptake occurs. In the "partial length uptake scenario" we consider that only active hyphae are taking up phosphate. The proportion of active hyphae is presented by an additional parameter $\phi$ so that the sink term $F_{\text {myc }}$ becomes

$F_{\text {myc }}(x, t, \rho, n, c)=2 \pi r_{\mathrm{h}} \phi \rho(x, t) \frac{F_{\mathrm{m}, \mathrm{h}} c(x, t)}{K_{\mathrm{m}, \mathrm{h}}+c(x, t)}$,

where $\phi$ has a value between 0 and 1 . When $\phi=0$ then hyphae are not taking up any $\mathrm{P}$, and when $\phi=1$ then hyphae are taking up $\mathrm{P}$ along their entire length. "Full length uptake scenario" and "tip uptake scenario" represent the most optimistic and the most conservative assumptions with respect to fungal $\mathrm{P}$ uptake. They are depicted in Fig. 3. "Partial length uptake scenario" is expected to lie somewhere in between.

\section{Parameterisation}

Apart from the new parameters in the sink terms of the tip and partial length uptake scenarios, the model is parameterised for $\mathrm{P}$ uptake from soil with literature data already used by Schnepf and Roose (2006) and Schnepf et al. (2007). The parameter values for $\mathrm{P}$ 
Fig. 3 Schematic representation of two uptake scenarios considered in this study: a uptake occurs along the entire hyphal length, and $\mathbf{b}$ uptake is restricted to a small zone at and behind the hyphal tips

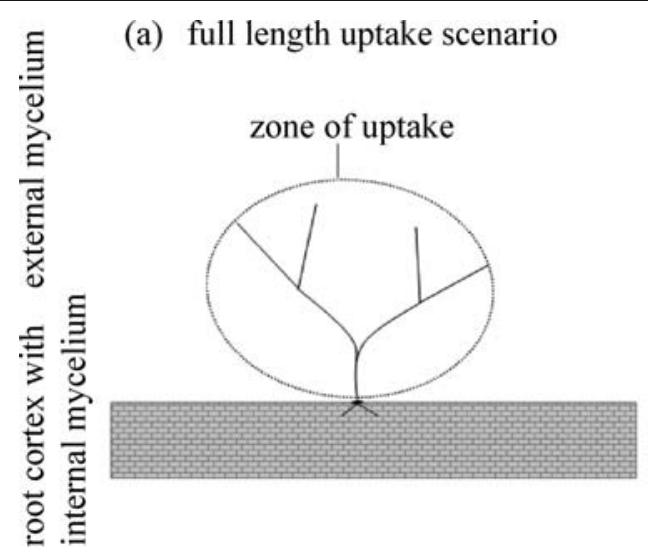

(b) tip uptake scenario

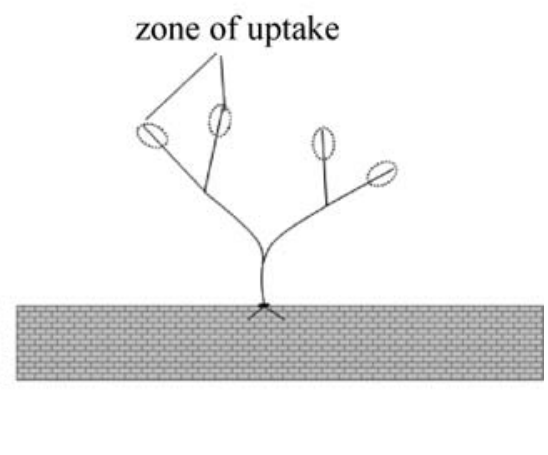

transport in soil are given in Table 1, the parameter values for $\mathrm{P}$ uptake into hyphae are given in Table 2. The fungal growth parameter values in Table 3 were obtained by calibrating the fungal growth model of Schnepf et al. (2007) to measured length densities of the external hyphae for three AM fungi described in Jakobsen et al. (1992a). The different growth patterns linear branching, nonlinear branching and anastomosis are expected to result in different soil depletion profiles and plant uptake. Additional differences are expected due to the different approaches with regard to the sites of hyphal $\mathrm{P}$ uptake.

The sink term $F_{\text {myc }}$ in the tip uptake scenario requires a value for the length of the zone of uptake near the hyphal tip. Jolicoeur et al. (1998) suggested that the zone of uptake has a width of $50-300 \mu \mathrm{m}$ behind all tips. In order to take the most conservative approach, we will use a value of $50 \mu \mathrm{m}$ in the following simulations. It is difficult to specify the parameter value for the proportion of active hyphae required in the partial length uptake scenario where the sink term is described by Eq. 11. To our knowledge, there are no available data that describe the spread of hyphae and at the same time distinguish between active and inactive hyphae. Histochemical staining of enzymes such as alkaline phosphatases has been used as an indicator of fungal viability or metabolic activity (Joner et al. 2000). As a result, part of the extraradical mycelium (given in percentage of the total hyphal length) is viewed as the active part of the mycelium and the rest is viewed as the inactive part of the mycelium. Although different enzymes are associated with different metabolic activities related to internal $\mathrm{P}$ transformations, these data indicate that often only part of the mycelium is involved in $\mathrm{P}$ uptake and translocation (Boddington and Dodd 1999). When we try to use this information in our model, we have to note that these numbers are not spatially explicit. In a first step, we will therefore estimate, combining information from the work of Boddington and Dodd (1999), Ezawa et al. (2003) and Bago (2000) that $50 \%$ of the mycelium at any given time and at any given distance from the root consists of active hyphae, i.e., in our model we choose $\phi=0.5$. Thus, from now on, we call the partial uptake scenario "half length uptake scenario".

Table 3 Parameter values for three fungal growth patterns as found in Schnepf et al. (2007)

\begin{tabular}{llllll}
\hline Symbol & Name & Units & $\begin{array}{l}\text { Linear branching } \\
\text { pattern }\end{array}$ & $\begin{array}{l}\text { Nonlinear branching } \\
\text { pattern }\end{array}$ & $\begin{array}{l}\text { Anastomosis } \\
\text { pattern }\end{array}$ \\
\hline$v$ & Elongation rate & $\mathrm{cm} \mathrm{s}^{-1}$ & $2.89 \times 10^{-6}$ & $1.50 \times 10^{-6}$ & $3.24 \times 10^{-6}$ \\
$b_{n}$ & Branching rate & $\mathrm{s}^{-1}$ & $2.31 \times 10^{-7}$ & $2.21 \times 10^{-5}$ & $1.70 \times 10^{-5}$ \\
$d_{n}$ & Tip death rate & $\mathrm{s}^{-1}$ & $6.02 \times 10^{-8}$ & $1.74 \times 10^{-6}$ & $8.45 \times 10^{-6}$ \\
$d$ & Hyphal death rate & $\mathrm{s}^{-1}$ & $2.08 \times 10^{-6}$ & $3.24 \times 10^{-2}$ & $9.26 \times 10^{-7}$ \\
$n_{\text {max }}$ & Inverse of the maximal tip density & $\mathrm{cm}^{-3}$ & 0 & $2.24 \times 10^{3}$ & $1.79 \times 10^{5}$ \\
$a_{1}$ & Tip-tip anastomosis rate & $\mathrm{cm}^{3} \mathrm{~s}^{-1}$ & 0 & 0 & $8.10 \times 10^{-12}$ \\
$a_{2}$ & Tip-side anastomosis rate & $\mathrm{cm}^{3} \mathrm{~cm}^{-1} \mathrm{~s}^{-1}$ & 0 & 0 & $1.74 \times 10^{-8}$ \\
$a$ & Root-soil boundary proliferation rate & $\mathrm{cm} \mathrm{cm}^{-3} \mathrm{~s}^{-1}$ & $2.92 \times 10^{-3}$ & $2.59 \times 10^{-3}$ & $6.48 \times 10^{-4}$ \\
$n_{0, b}$ & Initial tip density at root-soil boundary & $\mathrm{cm}^{-3}$ & 97.07 & 84.16 & 12.68 \\
\hline
\end{tabular}


Numerical solution

The model for P uptake by an arbuscular mycorrhizal root associated with one of three arbuscular mycorrhizal fungi with different growth pattern was solved numerically using the finite difference method (Morton and Mayers 1994). Specifically, an implicit finite difference scheme with centred differences in space was used to solve the model for P transport in soil given by Eqs. 1-4. The hyphal growth model given by Eqs. 6-9 was solved numerically using a LaxWendroff scheme for hyphal tip density and an explicit Euler scheme for the hyphal length density. All numerical schemes are described in detail in Schnepf and Roose (2006) and Schnepf et al. (2007).

\section{Results}

In this section we present results of the model computations for all combinations of the three different fungal growth patterns and the three different uptake scenarios. Model results include hyphal length and tip densities, $\mathrm{P}$ depletion profiles in soil, spatial distribution of $\mathrm{P}$ uptake by external hyphae, and the relative contributions of root and hyphae to overall $\mathrm{P}$ uptake, as well as an indication for the effectiveness of the mycelium.

Hyphal length and tip densities

In Fig. 4, the hyphal length densities of three AM fungi with different growth patterns after 4, 10 and 21 days are presented. Figure 4a shows the hyphal length density for the linear growth pattern where growth is based on linear branching with a low branching rate and a large supply of hyphal tips from the root surface. In this case, hyphal length density is largest near the root surface. The fungus shown in Fig. $4 \mathrm{~b}$ grows according to nonlinear branching pattern, i.e., branching ceases at a maximal hyphal tip density. From a biological point of view, this could be the result of competition for resources when the tip density becomes large. Figure $4 c$ shows the hyphal length density when growth follows the anastomosis pattern. It features a characteristic peak in hyphal length density near the front of maximum mycelial spread. In Fig. 5, the tip densities of the three fungal species are presented.

\section{Phosphate depletion in soil}

Figure 6 shows soil $\mathrm{P}$ depletion profiles away from the root surface due to both root and AM fungal uptake. Each row corresponds to one of the three uptake scenarios considered, and each column corresponds to one of the three growth patterns. For the linear and nonlinear fungal growth patterns in the full and half length uptake scenarios, the mycelium depletes the soil strongly as it grows away from the root surface. Depletion in the tip uptake scenario is significantly less than in the two other scenarios because there is a much smaller surface area available for uptake. For the anastomosis pattern in all uptake scenarios, the very different shape of the mycelial and hyphal tip distributions (Figs. $4 \mathrm{c}$ and $5 \mathrm{c}$ ) reflects in the shape of the modelled depletion zones (Fig. 6).

We assessed the amount of $\mathrm{P}$ still left in the soil region where the mycelium is present after 21 days by integrating $\mathrm{P}$ concentration profiles shown in Fig. 6 over the domain of the mycelium. In the full length uptake scenario, 13\% (anastomosis growth pattern),
Fig. 4 Hyphal length densities of three fungi with different growth patterns: a linear branching, b nonlinear branching, and $\mathbf{c}$ anastomosis

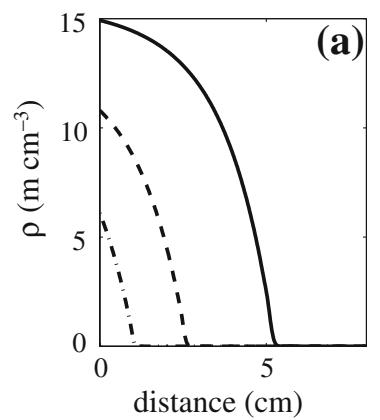

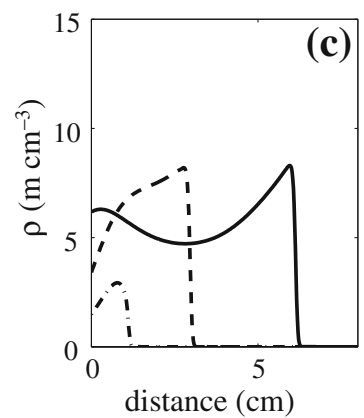

21 days - - - 10 days : - - - 4 days 
Fig. 5 Hyphal tip densities of three fungi with different growth patterns: a linear branching, b nonlinear branching, and $\mathbf{c}$ anastomosis

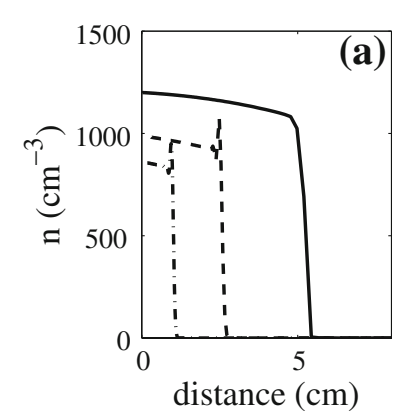

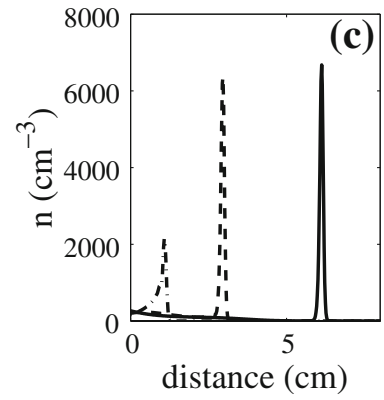

-21 days - - - 10 days $\quad---4$ days

$32 \%$ (linear branching growth pattern) and 57\% (nonlinear branching growth pattern) of the amount of $\mathrm{P}$ assumed to initially having been present in the soil solution are still left on day 21 . In the half length uptake scenario, 26\% (anastomosis), 40\% (linear branching) and 63\% (nonlinear branching) of the initial $\mathrm{P}$ content are still left. In the tip uptake scenario, depletion by the fungal mycelium is small, $97 \%$ of the initial $\mathrm{P}$ content are left by all fungal growth patterns. Differences between growth patterns are only in the spatial $\mathrm{P}$ acquisition but not in the overall effect.

\section{Distribution of mycelial P uptake rate}

Figure 7 shows the mycelial $\mathrm{P}$ uptake rate per unit volume of soil with respect to distance from the root surface assed from the sink term $F_{\text {myc }}$ of Eq. 1 . Uptake in the tip uptake scenario is lower by a factor 50-100 than uptake in the full and half length uptake scenarios. In the full and half length uptake scenarios, $\mathrm{P}$ is mainly taken up near the front of the spreading mycelium for all growth patterns. As it grows away from the root surface, it strongly depletes the soil and only the front of the mycelium grows into fresh,
Fig. $6 \mathrm{P}$ concentration in soil solution near a mycorrhizal root associated with AM fungi with different growth and uptake patterns: a linear branching/full length uptake, b nonlinear branching/full length uptake, c anastomosis/full length uptake, d linear branching/tip uptake, e nonlinear branching/tip uptake, f anastomosis/tip uptake, g linear branching/half length uptake, $\mathrm{g}$ nonlinear branching/half length uptake, $\mathbf{i}$ anastomosis/half length uptake
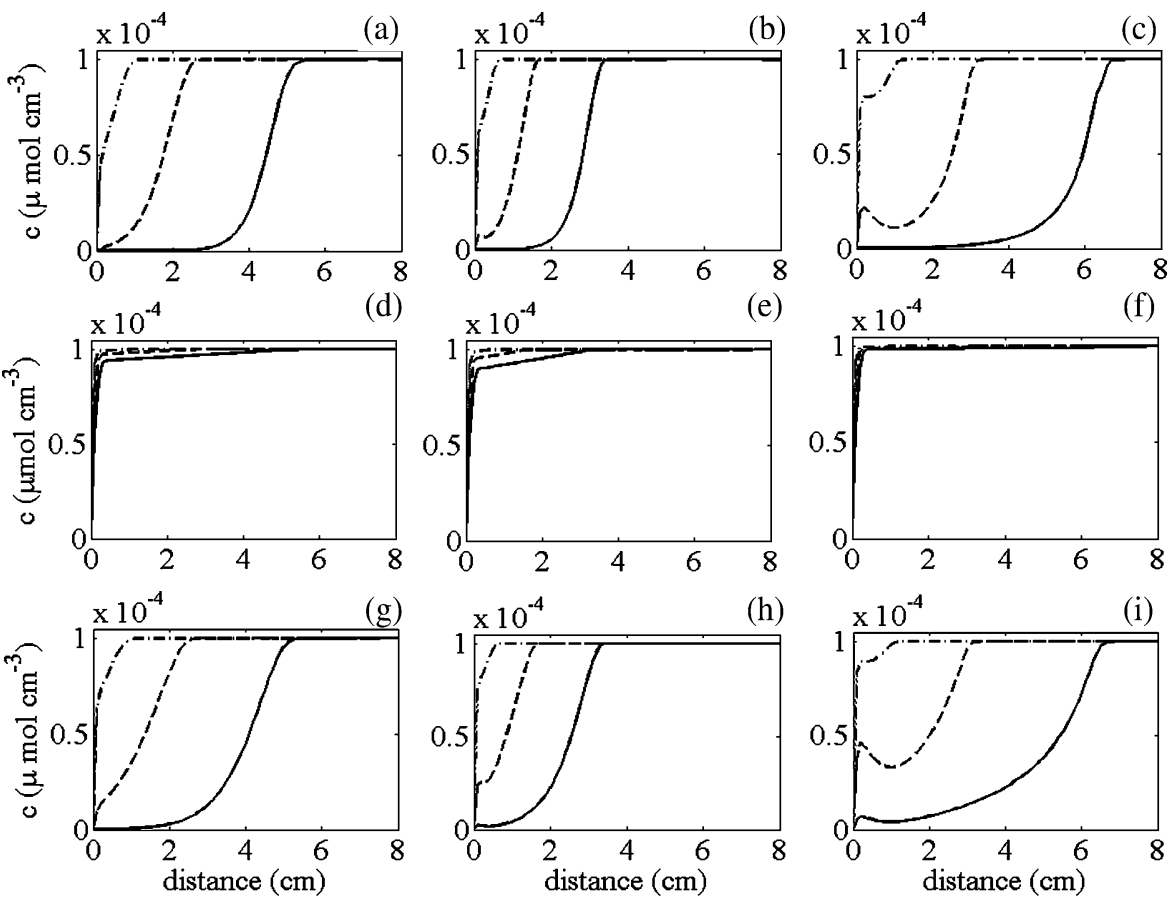

(h)
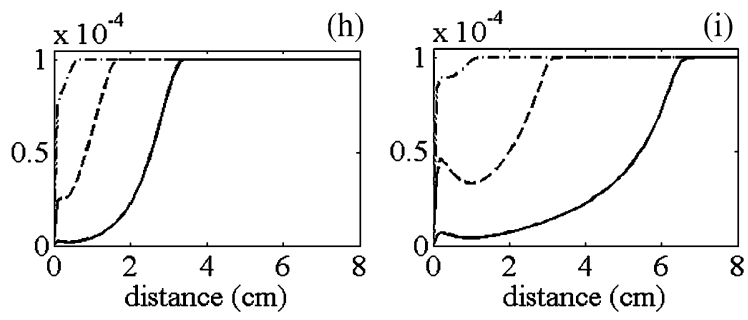

21 days ---10 days $-\cdot-\cdot 4$ days 
Fig. 7 Distribution of mycelial $\mathrm{P}$ uptake rate per unit volume of soil by AM fungi with different growth and uptake patterns: a linear branching/full length uptake, b nonlinear branching/ full length uptake, c anastomosis/full length uptake, d linear branching/tip uptake, e nonlinear branching/tip uptake, $\mathbf{f}$ anastomosis/tip uptake, g linear branching/ half length uptake, g nonlinear branching/half length uptake, $\mathbf{i}$ anastomosis/half length uptake
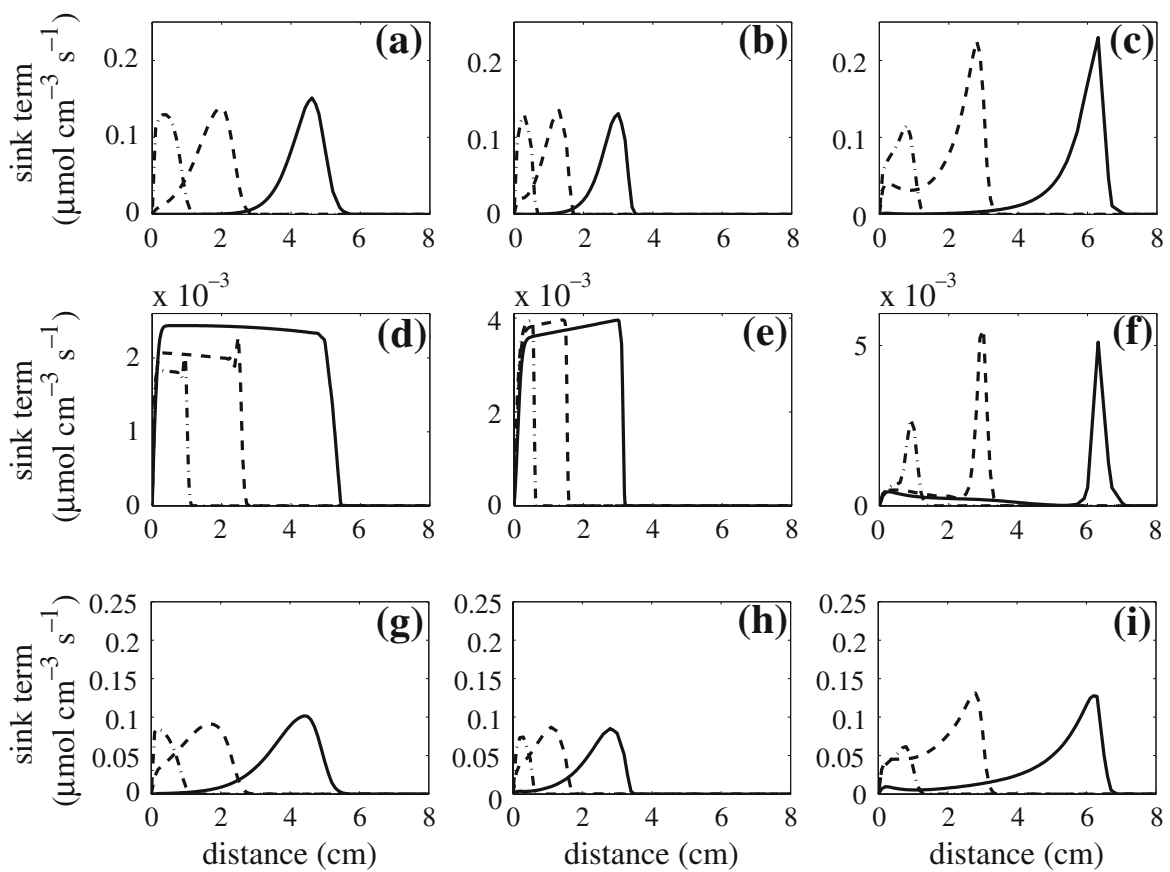

-21 days ---10 days $\cdots 4$ days

undepleted soil. In the tip uptake scenario, uptake by the fungus following the anastomosis pattern is also mostly occurring at the front of the colony. Contrary to this, uptake is more evenly distributed along the whole extension of the mycelium in the tip uptake scenario when the fungus grows by the linear or nonlinear branching pattern.

Phosphate influx into the root via the direct uptake pathway

Figure 8 shows the $\mathrm{P}$ influx into root which is due to the direct uptake pathway, on a double logarithmic scale, when the root is colonised with one of the three AM fungi with different growth patterns and for different uptake scenarios. In the full and half length uptake scenarios, the presence of the external hyphae reduces the influx of $\mathrm{P}$ into the root itself due to competition for $\mathrm{P}$ within the depletion zone created by the root. Among the different growth patterns, the fungus with the linear branching pattern has the largest hyphal length density at the root-soil boundary. This results in the fact that in the full and half length uptake scenarios this fungus reduces the root uptake most, followed by the fungus with the nonlinear branching and the anastomosis pattern. The situation is
Fig. 8 Root influx via the direct uptake pathway as influenced by competitive uptake by one of three fungal hyphae with different growth patterns: a full length uptake scenario, b tip uptake scenario, c half length uptake scenario

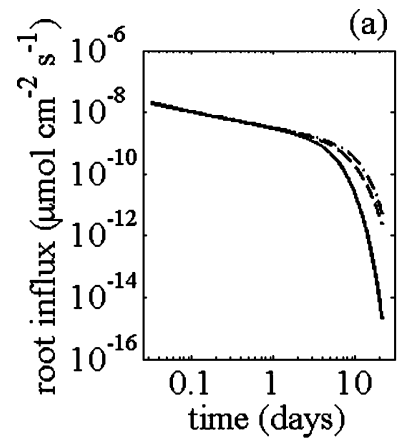

(a) $\widetilde{T} 10^{-6}$

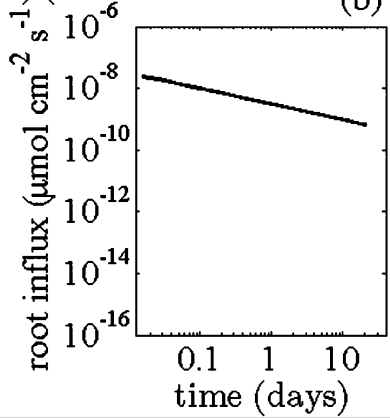

(b)

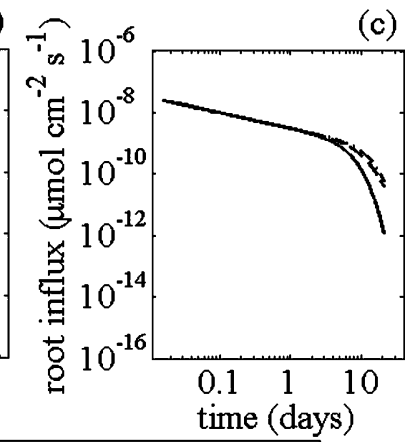

linear branching ----nonlinear branching ---.. anastomosis 
quite different in the tip uptake scenario where hyphae present almost no competition for root $\mathrm{P}$ uptake.

Relative contribution of fungal mycelium to overall $\mathrm{P}$ influx into a mycorrhizal root

The relative importance of hyphal versus overall influx into a mycorrhizal root is presented in Fig. 9. In the full and half length uptake scenarios, up to $100 \%$ of the overall influx is due to the fungal mycelium as soon as the mycelium is sufficiently large. The mycelium reaches the point where it is responsible for more than $50 \%$ of overall uptake within 2 days. Note that the relative contribution of mycelium to overall influx is the same in the full and half length scenarios. The overall influx however is less in the half length uptake scenario than in the full length uptake scenario. Among the different fungal growth patterns, the linear branching pattern is dominating overall influx faster than the other two species. This is not because it is more effective in uptake, but because it competes more with the root and hence root uptake is reduced (see Fig. 8 above and Fig. 10 below). In the tip uptake scenario, it takes more than 5 days until hyphae contribute to more than $50 \%$ of overall influx; the direct uptake pathway via roots is more significant. The contribution of mycelium with anastomosis growth pattern in particular is found to be below $70 \%$ of overall influx in the tip uptake scenario.

\section{Effectiveness of the fungal mycelium}

The plant has to invest carbon in order to support the fungal mycelium. It is therefore of interest, how effective this mycelium is with respect to its $\mathrm{P}$ uptake per unit fungal biomass. In this section, we compare the uptake rate by the entire fungal mycelium with its total hyphal length as a measure for the effectiveness of the mycelium. The effectiveness $E$ of mycelial uptake rate versus hyphal length of the mycelium at any given time is given by

$E(t)=\frac{\int_{0}^{x_{\mathrm{c}}} F_{\mathrm{myc}}(\rho, n, x, t) d x}{\int_{0}^{x_{\mathrm{c}}} \rho(x, t) d x}$,

where $x_{\mathrm{c}}$ is the position of the mycelial front. The results for all uptake scenarios are presented in Fig. 10. We use Eq. 12 only for times larger than 2 days, when the mycelium has been sufficiently established. Otherwise, when the total hyphal length is close to zero, we would have a singularity in Eq. 12. For all uptake scenarios and growth patterns, the effectiveness decreases with time because the fungal biomass increases whilst uptake decreases due to depletion. Because of this, some parts of the mycelium may be involved in transport processes inside the mycelium, but are not contributing to uptake. For all growth patterns, as expected, the mycelium is less effective in the half length uptake scenario than in the full length uptake scenario and more effective than in the tip uptake scenario. Table 4 shows the effectiveness for each fungal growth pattern at four different times relative to the most effective growth pattern within each of the different uptake scenarios. This expression reads $\frac{E(t)_{i}}{\max \left(E(t)_{i}\right)}$ where $i$ stands for the different fungal growth patterns. In the full and half length uptake scenarios, the fungus with the anastomosis pattern is most effective throughout the simulation period of 21 days (Fig. 10a, c; Table 4); second with respect to
Fig. 9 Influx due to hyphae relative to overall influx into a mycorrhizal root associated with one of three fungi with different growth patterns: a full length uptake scenario, b tip uptake scenario, c half length uptake scenario
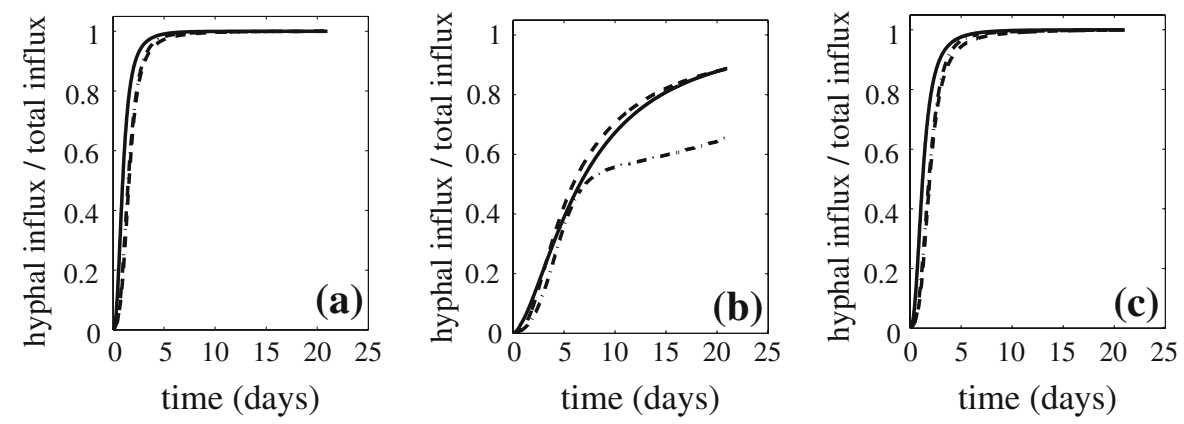

linear branching - - - nonlinear branching

- - - ' anastomosis 
Fig. 10 P uptake rate of the whole mycelium versus total hyphal length for three fungi with different growth patterns: a full length uptake scenario, b tip uptake scenario, c half length uptake scenario

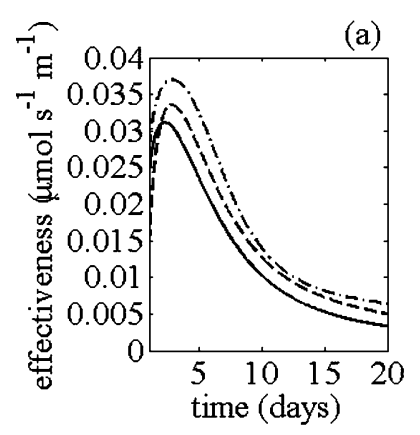

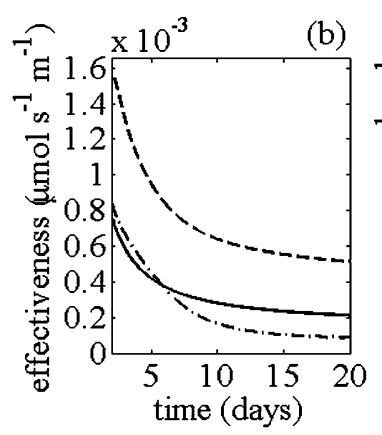

(c)

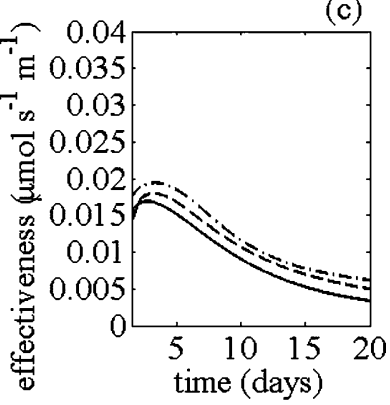

linear branching --- nonlinear branching $-\cdot-\cdot$ anastomosis

effectiveness is the fungus with the nonlinear branching pattern, followed by the one with the linear branching pattern. After 21 days, anastomosis pattern is twice as effective as the linear branching one (Table 4). In the tip uptake scenario, the fungus with the nonlinear branching pattern is significantly more effective than the two other patterns with the anastomosis one being the least effective in later times (Fig. 10b; Table 4). While the anastomosis pattern has the advantage that it creates a well interconnected mycelium, anastomosis also results in the loss of tips so that P uptake is reduced.

\section{Discussion}

This is the first mathematical model to quantify the effect of different AM fungal growth and uptake patterns on overall $\mathrm{P}$ influx into an arbuscular mycorrhizal root. It is a theoretical model study aimed to open the floor for a quantitative discussion about the processes involved in arbuscular mycorrhizal $\mathrm{P}$ uptake. Different conceptions about the processes involved in mycorrhizal $\mathrm{P}$ uptake that can be found in the literature

Table 4 Effectiveness of mycelium with respect to mycelial $\mathrm{P}$ uptake rate

$$
2 \text { days } 7 \text { days } 14 \text { days } 21 \text { days }
$$

Full and half length uptake scenarios

\begin{tabular}{lllll} 
Linear branching & 0.85 & 0.71 & 0.68 & 0.50 \\
Nonlinear branching & 0.88 & 0.85 & 0.91 & 0.78 \\
Anastomosis & 1 & 1 & 1 & 1 \\
Tip uptake scenario & & & & \\
Linear branching & 0.49 & 0.45 & 0.43 & 0.42 \\
Nonlinear branching & 1.00 & 1.00 & 1.00 & 1.00 \\
Anastomosis & 0.54 & 0.38 & 0.20 & 0.17 \\
\hline
\end{tabular}

are mechanistically represented in the model. Simulated soil depletion and $\mathrm{P}$ influx into a mycorrhizal root are largely different between the different scenarios. In order to verify or decline any of the hypotheses underlying these scenarios, the model needs to be validated with experimental data. While the modelling results obtained in the present study are in good agreement with data from a number of experimental studies in the literature, extrapolation to other AM fungal isolates of the species considered for the modelling here or to other AM fungal species and other experimental conditions has to be done with great caution. Using the model presented in this paper, we examined (a) the spatial acquisition of $\mathrm{P}$ by hyphae of arbuscular mycorrhizal fungi with different growth patterns, (b) the contribution of these hyphae to overall P influx into an arbuscular mycorrhizal root, and (c) assessed whether differences in uptake can be attributed to average structural features such as hyphal length densities.

Comparing modelled soil depletion with literature results

Model results shown in Fig. 6 were in good agreement with experimental findings that mycorrhizal roots develop a depletion zone beyond the zone of root influence (Joner et al. 1995; Li et al. 1991a; Smith et al. 2000). Simulated depletion profiles for the full and half length uptake scenarios show that a fungus with an anastomosis growth pattern leaves a higher $\mathrm{P}$ concentration near the root surface while the fungi with the linear and nonlinear branching patterns deplete the soil in a more uniform way. The latter is qualitatively similar to the results of Li et al. (1991a) who measured a uniform $\mathrm{P}$ depletion in the hyphal compartment by Glomus mosseae associated with white clover. Joner et al. (1995) measured overall 
depletion of $\mathrm{P}$ from a hyphal compartment in an experiment with cucumber associated with either Glomus invermaium or Glomus caledonium. They found that the soil in the hyphal compartment of $1 \mathrm{~cm}$ width was depleted to $52-66 \%$ of the initial $\mathrm{NaHCO}_{3}$ extractable $\mathrm{P}$ content. In $\mathrm{Li}$ et al. (1991b), the $\mathrm{NaHCO}_{3}$-extractable $\mathrm{P}$ in a hyphal compartment of $2 \mathrm{~cm}$ width was depleted to approximately $25 \%$ of the initial value by Glomus mosseae associated with white clover. These literature values are not directly comparable with model results on soil $\mathrm{P}$ depletion because they have been measured in confined hyphal compartments of limited width. However, they suggest that the full and half length uptake scenarios with depletion between $13-66 \%$ of the initial content in the soil zone that contains hyphae are closer to experimentally observed results than the tip uptake scenario where the soil containing hyphae was only depleted to $97 \%$ of the initial P content.

Comparing P inflow into a mycorrhizal root with literature results

Root inflow of P per unit length of root is calculated by multiplying root influx per unit root surface area with the root circumference. However, since our model is based on a planar root-soil interface, we can only estimate $\mathrm{P}$ inflow into a cylindrical root by assuming a value for the radius. Assuming a root radius of $0.2 \mathrm{~mm}$, simulated root $\mathrm{P}$ inflow ranged between 15.08 and $1,118.41 \mathrm{fmol} \mathrm{m}^{-1} \mathrm{~s}^{-1}$. Jakobsen et al. (1992a) give values of $P$ inflow of 122-2,056 fmol $\mathrm{m}^{-1} \mathrm{~s}^{-1}$. Published values of root $\mathrm{P}$ inflow into mycorrhizal plants on a whole-plant basis range from 300 to $12,000 \mathrm{fmol} \mathrm{m}^{-1} \mathrm{~s}^{-1}$ for mycorrhizal plants (Tinker and Nye 2000). However, one has to keep in mind that these experimentally obtained values for $\mathrm{P}$ inflow are generally obtained on a whole plant basis while or model results are on a single root basis. Methods for upscaling the model from single root to whole root system scale will enable more accurate comparisons between $\mathrm{P}$ inflow values measured on a whole root basis with simulated ones.

Spatial $\mathrm{P}$ acquisition by external mycelium

$\mathrm{P}$ uptake rate per unit volume of soil due to the external mycelium at different distances from the root surface and at different times as shown in Fig. 7 allows assessing the spatial acquisition of P. Model results for the linear and nonlinear branching patterns in the tip uptake scenario show uniform $\mathrm{P}$ uptake rate over the whole colony. In contrast to this, model results of the full and half length uptake scenarios and also for the tip uptake scenario when exhibiting an anastomosis growth pattern, $\mathrm{P}$ uptake rate due to the AM fungi occurs mostly near the front of the spreading mycelium. This effect is particularly pronounced for the fungal species with the anastomosis pattern as the hyphal length density is highest at the mycelial front. This suggests that the anastomosis growth pattern would be the most effective fungal growth pattern in order to access $\mathrm{P}$ from a distance. Indeed, the Acaulospora laevis isolate used by Jakobsen et al. (1992a) that exhibited the anastomosis growth pattern was shown to be a particular good symbiont to access $\mathrm{P}$ from a distance as they were able to show in a follow-up study (Jakobsen et al. 1992b). The other two fungal growth patterns result in a higher hyphal length density near the root and thus hyphae compete with the root for P. Differences between AM fungi in spatial $P$ uptake relative to the root surface are known (Drew et al. 2003; Jakobsen et al. 1992b; Jansa et al. 2005). So did Pearson and Jakobsen (1993) find that a strain of Glomus caledonium was most effective in accessing $\mathrm{P}$ from a distance compared to other fungal species. Smith et al. (2000) showed that a strain of $S$. calospora was less efficient to get ${ }^{33} \mathrm{P}$ from outside a root compartment than G. caledonium, but both fungi were equally effective with regard to total $\mathrm{P}$ uptake. Our model results could not reproduce experimental findings of Pearson and Jakobsen (1993) who found that the hyphal contribution to total $\mathrm{P}$ uptake of cucumber was small for Scutellospora calospora, but that root $\mathrm{P}$ uptake was improved due to root colonisation by $S$. calospora. Such a mechanism is currently not included in our model. We assume that differences in spatial $\mathrm{P}$ acquisition are solely attributable to the shape of the extraradical mycelium and the competition between roots and fungi. We did not include feedback mechanisms between AM root colonisation and $\mathrm{P}$ uptake on the one hand and root $\mathrm{P}$ uptake on the other hand. Such feedback mechanisms have however been suggested recently (Bucher 2007; Smith et al. 2004). Experimental data to quantify these feedback mechanisms are required in order to develop and parameterise such a model. 
Relative contribution of external mycelium to overall influx

The relative contributions of external mycelium and root to overall modelled $\mathrm{P}$ influx are shown in Fig. 9. $\mathrm{P}$ influx into the mycorrhizal root is dominated by the fungal mycelium in all uptake scenarios. However, in the tip uptake scenario, and in particular for the anastomosis growth pattern, roots contribute more considerably to overall influx. This means that in the full and half length uptake scenarios, we observe a "deactivation" of the root uptake pathway as suggested by Smith et al. (2003, 2004). Several reasons may be proposed for a massive reduction in direct $\mathrm{P}$ uptake into roots colonised by arbuscular mycorrhizal fungi. For example, the activity of $\mathrm{P}$ transporters located in root epidermal cells could be reduced as a response to AM fungal root colonisation (Smith et al. 2004). Under such a scenario, the root would not be able to take up $\mathrm{P}$ even if there was some available at the root surface. Although transporters are undoubtedly important, we observe this behaviour in our model results just by including P transport in soil and simultaneous uptake by AM fungal mycelium. Our results imply that extensive $\mathrm{P}$ depletion near the root due to the fungi may "deactivate" the direct uptake pathway via roots. In this model study this was the case for the linear and nonlinear branching patterns in the full and half length uptake scenarios.

Conclusions and outlook

The different fungal uptake scenarios resulted in large differences between soil $\mathrm{P}$ depletion and $\mathrm{P}$ influx into a mycorrhizal root. The different fungal growth patterns resulted in differences in the spatial $\mathrm{P}$ acquisition by the fungal mycelium. Comparing model output with literature data suggests that the half length uptake scenario agrees best with experimental findings. The tip uptake scenario clearly underestimates soil $\mathrm{P}$ depletion due to the fungal mycelium and the full length uptake scenario seems to overestimate it. While our model results currently suggest that the tip uptake scenario is less efficient than the other two scenarios, including additional mechanisms happening at the tips, such as solubilising fixed forms of soil $\mathrm{P}$, would probably consider this strategy more efficient.

With the help of still to be obtained experimental data on the dynamics of spread of AM fungal mycelia and the proportion of the mycelium actively involved in $\mathrm{P}$ uptake a more mechanistic approach for modelling the spread of P-absorbing active hyphae could be developed. Boswell et al. (2002) developed such a model for mycelial, but non-mycorrhizal, fungi. We suggest that this model framework could be adopted for arbuscular mycorrhizal fungi. With regard to the model equations, it is not much different from the fungal growth model presented in this paper. The fungal growth model described by Eqs. 6-9 simply needs to be changed in the following way. Firstly, we need to replace the variable $\rho$ by a new variable $\rho_{\text {active }}$ that represents the density of active hyphae. Secondly, the parameter $d$ in Eq. 6 can no longer be interpreted as the hyphal death rate, but taken to be the hyphal "inactivation rate". Thirdly, we need to assume that hyphae are first inactivated and then inactive hyphae may degrade into the environment. We describe the density of inactive hyphae with $\rho_{\text {inactive. }}$ This leads to the following model equations

$\frac{\partial \rho_{\text {active }}}{\partial t}=v n-d \rho_{\text {active }}$,

$\frac{\partial \rho_{\text {inactive }}}{\partial t}=d \rho_{\text {active }}-r \rho_{\text {inactive }}$

$\frac{\partial n}{\partial t}=-v \frac{\partial n}{\partial x}+b_{n}\left(1-\frac{n}{n_{\max }}\right)-d_{n} n-a_{1} n \rho_{\text {active }}-a_{2} n^{2}$,

$n=a t+n_{0, b}$, at $x=0, t>0$,

$\rho_{\text {active }}=\rho_{\text {inactive }}=n=0$, at $t=0$,

where $r$ is the degradation rate of inactive hyphae. One could also add additional hyphal death into Eq. 13 to allow that active hyphae can either be deactivated or die directly.

The model presented in this paper could further be extended in order to explicitly model the plant carbon costs. The whole symbiosis including the bidirectional transfer of carbon and $\mathrm{P}$, respectively, can be modelled. More experimental data to parameterise and validate such models are required. A more 
complex description of the architecture of the extraradical mycelium could for example distinguish between "runner hyphae", the skeleton of the fungal colony, and "branched adsorbing structures" (BAS), small bunches of dichotomously branched hyphae formed by second and higher orders of runner hyphae (Bago et al. 1998). Bago (2000) suggested that BAS are the preferential site of hyphal P uptake. Including the findings of such morphological studies into the growth model for the external mycelium of AM fungi might lead to different simulated depletion zones and $\mathrm{P}$ uptake. However, the extension of the present fungal growth model to include BAS would require substantial changes to the model structure and we will address this in our future work. Further model extensions are related to the competition between several mycorrhizal roots and upscaling this single root model to the root system scale. Ongoing collaboration between modellers and experimentalists is currently examining mathematical multiscale methods for the purpose of upscaling from the single root to the root system scale. We expect that this work will also influence the upscaling of mycorrhizal roots.

Acknowledgements Andrea Schnepf is a Hertha-Firnberg Research Fellow (FWF Austrian Science Fund, Project T341N13). Tiina Roose is a Royal Society University Research Fellow. We acknowledge two anonymous reviewers whose comments have truly strengthened this paper.

Open Access This article is distributed under the terms of the Creative Commons Attribution Noncommercial License which permits any noncommercial use, distribution, and reproduction in any medium, provided the original author(s) and source are credited.

\section{References}

Bago B (2000) Putative sites for nutrient uptake in arbuscular mycorrhizal fungi. Plant Soil 226:263-274, doi:10.1023/ A:1026456818903

Bago B, Azcon-Aguilar C, Piche Y (1998) Architecture and developmental dynamics of the external mycelium of the arbuscular mycorrhizal fungus Glomus intraradices grown under monoxenic conditions. Mycologia 90:52-62, doi: $10.2307 / 3761011$

Barber SA (1995) Soil nutrient bioavailability: a mechanistic approach. Wiley, New York

Boddington CL, Dodd JC (1999) Evidence that differences in phosphate metabolism in mycorrhizas formed by species of Glomus and Gigaspora might be related to their lifecycle strategies. New Phytol 142:531-538, doi:10.1046/ j.1469-8137.1999.00422.x
Boswell GP, Jacobs H, Davidson FA, Gadd GM, Ritz K (2002) Functional consequences of nutrient translocation in mycelial fungi. J Theor Biol 217:459-477, doi:10.1006/ jtbi. 2002.3048

Brundrett MC (2002) Coevolution of roots and mycorrhizas of land plants. New Phytol 154:275-304, doi:10.1046/ j.1469-8137.2002.00397.x

Bucher M (2007) Functional biology of plant phosphate uptake at root and mycorrhiza interfaces. New Phytol 173:11-26, doi:10.1111/j.1469-8137.2006.01935.x

Drew EA, Murray RS, Smith SE, Jakobsen I (2003) Beyond the rhizosphere: growth and function of arbuscular mycorrizal external hyphae in sands of varying pore sizes. Plant Soil 251:105-114, doi:10.1023/A:1022932414788

Ezawa T, Smith SE, Smith FA (2002) P metabolism and transport in AM fungi. Plant Soil 244:221-230, doi:10. 1023/A:1020258325010

Ezawa T, Cavagnaro TR, Smith SE, Smith FA, Ohtomo R (2003) Rapid accumulation of polyphosphate in extraradical hyphae of an arbuscular mycorrhizal fungus as revealed by histochemistry and a polyphosphate kinase/ luciferase system. New Phytol 161:387-392, doi:10.1046/ j.1469-8137.2003.00966.x

Hinsinger P, Gobran GR, Gregory PJ, Wenzel WW (2005) Rhizosphere geometry and heterogeneity arising from rootmediated physical and chemical processes. New Phytol 168:293-303, doi:10.1111/j.1469-8137.2005.01512.x

Gisi U, Schenker R, Schulin R, Stadelmann F, Sticher H (1997) Bodenökologie. Georg Thieme Verlag, Stuttgart, New York

Jakobsen I, Abbott LK, Robson AD (1992a) External hyphae of vesicular-arbuscular mycorrhizal fungi associated with Trifolium subterraneum L. 1. Spread of hyphae and phosphorus inflow into roots. New Phytol 120:371-380, doi:10.1111/j.1469-8137.1992.tb01077.x

Jakobsen I, Abbott LK, Robson AD (1992b) External hyphae of vesicular-arbuscular mycorrhizal fungi associated with Trifolium subterraneum L. 2. Hyphal transport of ${ }^{32} \mathrm{P}$ over defined distances. New Phytol 120:509-516, doi:10.1111/ j.1469-8137.1992.tb01800.x

Jansa J, Mozafar A, Frossard E (2005) Phosphorus acquisition strategies within arbuscular mycorrhizal fungal community of a single field site. Plant Soil 276:163-176, doi:10. 1007/s11104-005-4274-0

Jolicoeur M, Germette S, Gaudette M, Perrier M, Bécard G (1998) Intracellular $\mathrm{pH}$ in arbuscular mycorrhizal fungi. Plant Physiol 115:1279-1288, doi:10.1104/pp.116.4. 1279

Joner EJ, Magid J, Gahoonia TS, Jakobsen I (1995) P depletion and activity of phosphatases in the rhizosphere of mycorrhizal and non-mycorrhizal cucumber (cucumis sativus L.). Soil Biol Biochem 27:1145-1151, doi:10. 1016/0038-0717(95)00046-H

Joner E, van Aarle I, Vosatka M (2000) Phosphatase activity of extra-radical arbuscular mycorrhizal hyphae: a review. Plant Soil 226:199-210, doi:10.1023/A:1026582 207192

Li X-L, George E, Marschner H (1991a) Extension of the phosphorus depletion zone in VA-mycorrhizal white clover in a calcareous soil. Plant Soil 136:41-48, doi:10.1007/BF02465218 
Li X-L, George E, Marschner H (1991b) Phosphorus depletion and $\mathrm{pH}$ decrease at the root-soil and hyphae-soil intefaces of VA mycorrhizal white clover fertilized with ammonium. New Phytol 119:397-404, doi:10.1111/j.1469-8137.1991. tb00039.x

Lide DR (2000) Handbook of chemistry and physics. CRC, Boca Raton, FL

Morton KW, Mayers DF (1994) Numerical solution of partial differential equations. Cambridge University Press, New York, NY

Pearson JN, Jakobsen I (1993) The relative contribution of hyphae and roots to phosphorus uptake by arbuscular mycorrhizal plants, measured by dual labelling with ${ }^{32} \mathrm{P}$ and ${ }^{33} \mathrm{P}$. New Phytol 124:489-494, doi:10.1111/j.14698137.1993.tb03840.x

Raghothama KG, Karthikeyan AS (2005) Phosphate acquisition. Plant Soil 274:37-49, doi:10.1007/s11104-004-2005-6

Schnepf A, Roose T (2006) Modelling the contribution of arbuscular mycorrhizal fungi to plant phosphate uptake. New Phytol 171:669-682

Schnepf A, Roose T, Schweiger P (2007) Growth model for arbuscular mycorrhizal fungi. J R Soc Interface 5:773784, doi:10.1098/rsif.2007.1250

Schweiger P, Jakobsen I (1999) The role of mycorrhizas in plant $\mathrm{P}$ nutrition: fungal uptake kinetics and genotype variation. In: Gissel-Nielsen G, Jensen A (eds) Plant nutrition-molecular biology and genetics. Kluwer Academic, Dordrecht, pp 277-289

Smith FA, Jakobsen I, Smith SE (2000) Spatial differences in acquisition of soil phosphate between two arbuscular mycorrhizal fungi in symbiosis with Medicago truncatula. New Phytol 147:357-366, doi:10.1046/j.1469-8137. 2000.00695.x

Smith SE, Smith FA, Jakobsen I (2003) Mycorrhizal fungi can dominate phosphate supply to plants irrespective of growth responses. Plant Physiol 130:16-20, doi:10.1104/ pp. 103.024380

Smith SE, Smith FA, Jakobsen I (2004) Functional diversity in arbuscular mycorrhizal (AM) symbioses: the contribution of the mycorrhizal P uptake pathway is not correlated with mycorrhizal responses in growth or total $\mathrm{P}$ uptake. New Phytol 162:511-524, doi:10.1111/j.1469-8137.2004.01039.x

Tinker TB, Nye PH (2000) Solute movement in the rhizosphere. Oxford University Press, Oxford

Vance CP (2003) Phosphorus acquisition and use: critical adaptations by plants for securing a nonrenewable resource. New Phytol 157:423-447, doi:10.1046/j.14698137.2003.00695.x

Zhu YG, Cavagnaro TR, Smith SE, Dickson S (2001) Backseat driving? Accessing phosphate beyond the rhizospheredepletion zone. Trends Plant Sci 6:194-195, doi:10.1016/ S1360-1385(01)01957-4 